\section{Diabetes Mellitus tipo 2 con tendencia a la cetosis. Caso clínico}

\author{
LUCIANA CONCHA L. ${ }^{1}$, PILAR DURRUTY A. ${ }^{2,3, a}$, \\ MANUEL GARCÍA DE LOS RÍOS A. ${ }^{2}$
}

\section{Ketosis prone type 2 diabetes (KPD)}

Ketosis prone type 2 diabetes (KPD) is presently a well-defined clinical entity, characterized by a debut with severe hyperglycemia and ketoacidosis similar to the presenting form of Type 1 diabetes mellitus (DM1). However, it appears in subjects with Type 2 diabetes mellitus (DM2) phenotype. This situation is caused by an acute, reversible dysfunction of the beta cell in individuals with insulin resistance. Once the acute stage subsides, patients behave as having a DM2 and do not require insulin treatment. They should be kept on a diet and oral hypoglycemic drugs due to their susceptibility to have recurrent acute ketotic decompensations.

(Rev Med Chile 2015; 143: 1215-1218)

Key words: Type 2 diabetes mellitus; Hypoglycemic agents; Insulin; Ketosis.
'Unidad de Endocrinología y Diabetes. Hospital Gustavo Fricke, Viña del Mar, Servicio y Departamento de Medicina. Facultad de Medicina Universidad de Valparaíso.

${ }^{2}$ Unidad de Diabetes, Hospital San Juan de Dios, Servicio y Departamento de Medicina Occidente. Facultad de Medicina Universidad de Chile.

${ }^{3}$ Servicio de Endocrinología y Diabetes, Hospital Clínico Universidad de Chile. aBioquímico.

Sin financiamiento. No hay conflicto de interés.

Recibido el 5 de mayo de 2015, aceptado el 27 de julio de 2015.

Correspondencia a: Luciana Concha L. Los Jazmines 894, Casablanca, V Región, Chile.

luciana.concha@hotmail.com
L a mayoría de los pacientes con diabetes mellitus (DM) se pueden clasificar en uno de los cuatro grupos actualmente aceptados ${ }^{1,2}$ teniendo en cuenta la heterogeneidad de cada uno de ellos. Sin embargo, 10\% de los casos presenta algún grado de dificultad en su tipificación. Se trata principalmente de adultos jóvenes que inician su enfermedad como diabetes mellitus tipo 1 (DM1), pero que evolucionan como diabetes mellitus tipo 2 (DM2). Este "subtipo" de DM se ha denominado diabetes mellitus tipo 2 con tendencia a la cetosis (DTC).

\section{Caso clínico}

Hombre de 45 años, $80 \mathrm{~kg}$, talla 1,72 m, IMC $27,11 \mathrm{~kg} / \mathrm{m}^{2}$, portador de dislipidemia, sin antecedentes familiares de DM. Inicia un cuadro caracterizado por polidipsia, poliuria, cansancio y calambres. Al mes de evolución consulta en un servicio de urgencia. En el examen físico destacan piel y mucosas secas, acantosis nigricans en cuello, normotenso y pérdida de 8 kilos de peso.

Se realizan exámenes (Tabla 1) y se hospitaliza con el diagnóstico de cetoacidosis diabética
(CAD). Se inicia solución solución salina $0,9 \%$ $1.000 \mathrm{cc}$ con cloruro de potasio e insulina rápida (IR) $8 \mathrm{U}$ endovenosa. A la hora, se continúa con el aporte de volumen $(3.000 \mathrm{cc})$ e IR sc (subcutánea) cada 6 h, según glicemias capilares. Se descarta foco infeccioso. A las $8 \mathrm{~h}$ de tratamiento, al revertir la acidosis (Tabla 1) y lograr glicemias $<250$ $\mathrm{mg} / \mathrm{dl}$, se inicia terapia basal bolos con insulina NPH (Neutral Protamine Hagedorn) e IR antes del desayuno $(\mathrm{AD})$ y cena, más refuerzos de IR pre-prandiales, lográndose una buena respuesta clínica. $\mathrm{Al}$ alta se indica régimen con $180 \mathrm{~g}$ de hidratos de carbono, insulina NPH 18 U AD y $6 \mathrm{U}$ a las $22 \mathrm{~h}$, con automonitoreo de glicemias capilares.

A las 2 semanas el paciente se encuentra asintomático con glicemias capilares de ayunas 70-110 $\mathrm{mg} / \mathrm{dl}$, pre-prandiales $100-130 \mathrm{mg} / \mathrm{dl}$ y post-prandiales 160-180 mg/dl. Dada su buena evolución se disminuye la insulina NPH a $6 \mathrm{U}$ a las $22 \mathrm{~h}$ y se agrega metformina $850 \mathrm{mg} 2$ veces al día. Controlado al mes, relata episodios de hipoglicemias de $60 \mathrm{mg} / \mathrm{dl}$ durante el día y los exámenes revelan buen control metabólico (Tabla 2).

Dado el inicio de una DM en CAD (sospecha de DM1), en un paciente con características fenotípicas de DM2, se solicitaron marcadores 
Tabla 1. Niveles de parámetros bioquímicos, hemograma y ECG realizados en el Servicio de Urgencia

\begin{tabular}{|c|c|c|}
\hline & Ingreso & A las 8 h \\
\hline Glicemia (mg/dl) & 640 & 250 \\
\hline $\mathrm{pH}$ & 7,32 & 7,44 \\
\hline $\mathrm{HCO}^{*}(\mathrm{mEq} / \mathrm{l})$ & 20 & 24 \\
\hline Potasio $(\mathrm{mEq} / \mathrm{l})$ & 3,8 & 3,5 \\
\hline Sodio (mEq/l) & 140 & 142 \\
\hline Cloro (mEq/l) & 105 & 110 \\
\hline Cetonemia & +++ & + \\
\hline Anion gap (mEq/l) & 18 & 11,5 \\
\hline $\mathrm{HbA} 1 \mathrm{c}^{* *}(\%)$ & & 8,8 \\
\hline Creatinina (mg/dl) & 0,8 & 0,8 \\
\hline Colesterol total $(\mathrm{mg} / \mathrm{dl})$ & 200 & \\
\hline Colesterol HDL(mg/dl) & 40 & \\
\hline Colesterol LDL(mg/dl) & 94 & \\
\hline Triglicéridos(mg/dl) & 330 & \\
\hline $\begin{array}{l}\text { Orina } \\
\text { Glucosuria } \\
\text { Cetonuria }\end{array}$ & $\begin{array}{l}+++ \\
+++\end{array}$ & $\begin{array}{l}+ \\
+\end{array}$ \\
\hline Hemograma & Normal & Normal \\
\hline Electrocardiograma & Normal & \\
\hline
\end{tabular}

*HCO3 Bicarbonato de sodio. ** HbA1c Hemoglobina glicosilada A1c.

inmunológicos para diabetes: anticuerpos anti células beta (ICA), anticuerpos anti insulina (IAA), anticuerpos anti ácido glutámico descarboxilasa (anti-GAD), anticuerpos anti tirosina fosfatasa (IA2), los que resultaron negativos. El péptido $\mathrm{C}$ en ayunas fue normal $(3,2 \mathrm{ng} / \mathrm{ml})$. En estas condiciones se suspendió la insulina, manteniéndose las medidas no farmacológicas y la metformina.

El paciente continúa en controles periódicos durante 24 meses post alta, lográndose una reducción de $10 \mathrm{~kg}$ de peso, glicemias de ayunas inferiores a100 mg/dl, HbAlc 5,9\%, y perfil lipídico y presión arterial normales.

\section{Discusión}

El caso clínico que se comenta correspondería por su forma de presentación en CAD y posterior evolución propia de una DM2 con rápida respues-
Tabla 2. Niveles de glicemia, HbA1c, perfil lipídico, creatinina y orina realizados a las 6 semanas post alta

\begin{tabular}{|lc|}
\hline Glicemia $(\mathrm{mg} / \mathrm{dl})$ & 90 \\
\hline $\mathrm{HbA}_{1} \mathrm{c}^{*}(\%)$ & 7,2 \\
Colesterol total $(\mathrm{mg} / \mathrm{dl})$ & 180 \\
$\quad$ Colesterol HDL $(\mathrm{mg} / \mathrm{dl})$ & 44 \\
Colesterol LDL $(\mathrm{mg} / \mathrm{dl})$ & 96 \\
\hline Triglicéridos $(\mathrm{mg} / \mathrm{dl})$ & 200 \\
Creatinina $(\mathrm{mg} / \mathrm{dl})$ & 0,7 \\
Orina & Normal \\
\hline
\end{tabular}

*HbA1c Hemoglobina glicosilada A1c.

ta a la terapia oral, a una DTC, conocida en inglés como ketosis prone type 2 diabetes.

Este subtipo de DM es una entidad clínica única, no incorporada aún en la clasificación actual de la American Diabetes Association (ADA) ${ }^{1}$, ni de la World Health Organization/International Diabetes Federation (WHO/IDF) ${ }^{2}$, pese a que la primera referencia a este cuadro corresponde a 1987 en Estados Unidos de Norteamérica ${ }^{3}$, existiendo posteriormente numerosas publicaciones al respecto ${ }^{4,5}$. Esta variante de DM2 se había denominado diabetes atípica, diabetes intermedia, DM 1,5 o "Flatbush diabetes".

La DTC se ha descrito en Estados Unidos de Norteamérica principalmente en afroamericanos ${ }^{6}$ donde representaría entre $20-50 \%$ de los casos de DM en esta etnia. Actualmente la DTC es reconocida en todas las poblaciones. Así, en Norteamérica se ha informado que también se presenta en hispanos (20-50\%) y aproximadamente en 10\% de los asiáticos y blancos ${ }^{7,8}$. Nosotros publicamos en el año 2001 un grupo de pacientes que correspondería a este subtipo de DM2 ${ }^{9}$.

Por la dificultad que presenta para algunos casos la clasificación de diabetes actualmente utilizada ${ }^{10}$, se ha propuesto un esquema complementario denominado $A \beta^{11,12}$, que usa la presencia $o$ ausencia de autoanticuerpos (A+o A-) y de función beta celular (secreción de insulina), evaluada por péptido $C$, con o sin secreción $(\beta+o \beta-)$, que se realiza a las 3-6 semanas del cuadro agudo de $\mathrm{CAD}^{4}$. La mayoría de los pacientes con DTC serían $(A-\beta+)$, ya que tienen reserva insulínica, pero sin autoanticuerpos, lo que los diferenciaría de los DM1 que son $A+\beta-$. 
La etiopatogenia de la DTC es aún desconocida, aunque existen hechos destacables, con discrepancia en los hallazgos publicados. A estos pacientes se les ha descrito alteración en la secreción de insulina por una destrucción no autoinmune de las células beta, el grado de deficiencia insulínica es variable y los requerimientos de ésta son fluctuantes. Al inicio los DTC tienen niveles bajos de péptido $\mathrm{C}$, aunque en cifras que duplican los valores de los DM1 (0,21 vs $0,12 \mathrm{ng} / \mathrm{ml})$, pero $60 \%$ menos que los DM2. Se puede decir que la secreción de insulina en los DTC es intermedia entre los DM1 y los DM2. A los 10 años de enfermedad, tienen $60 \%$ de la producción normal de insulina, similar a los DM2 ${ }^{13}$.

Los casos de DTC presentan resistencia a la insulina en forma concomitante al déficit de secreción. Con el buen control metabólico se logra a los meses de evolución una mejoría de ambos ejes patogénicos, lo que permite suspender el tratamiento insulínico y manejar a los pacientes con metformina o sulfonilureas. Se deben mantener los hipoglicemiantes orales (HGO) para prevenir la recurrencia de la cetoacidosis ${ }^{14}$.

El principal determinante de la severa descompensación metabólica sería una falla transitoria de la función beta celular, debida probablemente a glucotoxicidad en individuos con RI, la que se revierte con la normalización de la glicemia ${ }^{15}$. La glucotoxicidad-hiperglicemia crónica sostenidaes un fenómeno que produce una mayor RI y una intensificación en la falla de secreción insulínica.

La lipotoxicidad también participaría en la falla beta celular y en la resistencia a la insulina. Se han postulado defectos en la utilización de ácidos grasos y cetogénesis acelerada como otro posible mecanismo patogénico de la $\mathrm{DTC}^{16}$. Umpierrez, en su publicación de 2010, no confirma esta hipótesis ${ }^{17}$.

Una teoría muy atractiva es la propuesta por Sobngwi et $\mathrm{a}^{18}$, quienes encontraron en los pacientes con DTC una alta prevalencia de deficiencia de la enzima glucosa 6-fosfato deshidrogenasa (G6PD) comparados con los controles y los DM2. Ciertos genes que controlan la secreción de insulina y las defensas antioxidantes a través de la G6PD, podrían predisponer a la diabetes con tendencia a la cetosis.

La cetosis/cetoacidosis ocurre en forma simultánea al déficit de insulina y aumento del glucagón. En este sentido, se ha informado que los pacientes con DTC tienen altos niveles basales de glucagón y la supresión de su liberación esta disminuida ${ }^{19}$, lo que sugiere que las células alfa podrían ser importantes en la patogenia de este subtipo de diabetes. Es sorprendente que en estos pacientes ocurre cetosis por una menor utilización de ácidos grasos libres y cetonas, más que por un aumento de la lipólisis y producción de cuerpos cetónicos ${ }^{12}$.

Clínicamente se ha descrito que la DTC es más frecuente en el sexo masculino (3:1), edad promedio 40 años (33-53 años) y en sujetos obesos con antecedentes familiares de DM2. La mayoría de los pacientes tienen un síndrome diabético agudo 4 a 6 semanas previas a su diagnóstico, y el examen físico frecuentemente muestra marcadores de RI (acantosis nigricans y obesidad abdominal). $\mathrm{Al}$ debut presentan características clínicas y parámetros bioquímicos y ácido base de una CAD, sin causa precipitante ${ }^{8}$. Se deben tratar de acuerdo a los protocolos recomendados para el manejo de la $\mathrm{CAD}^{20}$, con reposición agresiva de fluidos, insulinoterapia continua, vigilancia y tratamiento de los desórdenes electrolíticos y ácido base. Una vez resuelta la etapa aguda, se inicia manejo con insulina en esquemas basal-bolos.

Generalmente al alta continúan sólo con insulinas basales, lo que facilita la recuperación de la célula $\beta$ y mejora la sensibilidad a la insulina. Para minimizar los efectos de la glucotoxicidad y lipotoxicidad sobre la célula $\beta$, a las 3 a 6 semanas de ocurrido el evento agudo, se debería evaluar la reserva pancreática a través de la determinación de péptido $\mathrm{C}$ en ayunas y la presencia de autoinmunidad. En la mayoría de los pacientes con DTC $(A-\beta+)$ se logra suspender la insulina antes de los 6 meses ( 40 a $50 \%$ ), incluso a los 10 años $40 \%$ no la requiere nuevamente ${ }^{21}$.

En su evolución, al mantener a los pacientes con dieta exclusiva, 60 a 70\% tiene hiperglicemias a los 2 años. Por ello, al suspender la insulina, requieren iniciar o mantener el uso de $\mathrm{HGO}^{14}$, ya que tienden a presentar con mayor frecuencia que los DM2 descompensaciones metabólicas con cuadros de $\mathrm{CAD}$, debido a la labilidad beta celular que los caracteriza.

\section{Conclusión}

La DTC es un subtipo de DM2 bien identificada en los últimos años. Ha sido descrita en 
varios países incluido Chile. Su presentación y evolución natural es diferente a los DM1 y DM2 clásicos. El conocimiento de esta entidad permite un diagnóstico oportuno y tratamiento adecuado.

\section{Referencias}

1. Report of the Expert Committee on the Diagnosis and Classification of Diabetes Mellitus. Diabetes Care 1997; 20: 1183-97.

2. Report of a WHO/IDF consultation. Definition and diagnosis of diabetes mellitus and intermediate hyperglycemia. Geneva: World Health Organization 2006. http://www.who.int/diabetes/publications/diagnosis_diabetes2006/en/

3. Winter WE, Maclaren NK, Riley WJ, Clarke DW, Kappy MS, Spillar RP. Maturity-onset diabetes of youth in black Americans. N Engl J Med 1987; 31: 285-91.

4. Umpierrez GE. Ketosis-Prone Type 2 Diabetes. Time to revise the classification of diabetes. Diabetes Care 2006; 29: 2755-7.

5. Goodstein G, Milanesi A, Weinreb JE. Ketosis-Prone Type 2 Diabetes in a Veteran Population. Diabetes Care 2014; 37: e74-5.

6. Umpierrez G, Smiley D, Kitabchi A. Narrative Review: Ketosis-Prone Type 2 Diabetes Mellitus. Ann Intern Med 2006; 144: 350-7.

7. Colloby M. Ketosis-prone diabetes: Identification and management. Journal of Diabetes Nursing 2014; 18: 352-60.

8. Smiley D, Chandra P, Umpierrez G. Update on diagnosis, pathogenesis and management of ketosis prone Type 2 diabetes mellitus. Diabet Manag 2011; 1: 589600.

9. Raddatz V, Durruty P, Briones G, López G, Soto N, García de los Ríos M. Subtipos "no clásicos” de diabetes mellitus. Rev Med Chile 2001; 129: 853-60.

10. Stone MA, Camosso-Stefinovic J, Wilkinson J, De Lusignan S, Hattersley AT, Khunti K, et al. Incorrect and incomplete coding and classification of diabetes: a systematic review. Diabetic Med 2010; 27: 491-7.

11. Balasubramanyam A, Garza G, Rodríguez LVN, Hampe CS, Gaur L, Lenmark A, et al. Accuracy and predictive value of classification schemes for ketosis prone diabetes. Diabetes Care 2006; 29: 2575-9.

12. Maldonado M, Hampe CS, Gaur LK, D’Amico S, Iyer D, Hammerle LP, et al. Ketosis-prone diabetes: dissection of a heterogeneous syndrome using an immunogenetic and beta-cell functional classification, prospective analysis, and clinical outcomes. J Clin Endocrinol Metab 2003; 88 (11): 5090-8.

13. Mauvais-Jarvis F, Sobngwi E, Porcher R, Riveline JP, Kevorkian JP, Vaisse C, et al. Ketosis-prone type 2 diabetes in patients of sub-saharan African origin. Diabetes 2004; 53: 645-53.

14. Umpierrez GE, Clark WS, Steen MT. Sulfonylureas treatment prevents recurrence of hyperglycemia in obese African-American patients with a history of hyperglycemic crises. Diabetes Care 1997; 20: 479-83.

15. Gosmanov AR, Smiley D, Robalino G, Siqueira JM, Peng L, Kitabchi AE, et al. Effects of intravenous glucose load on insulin secretion in patients with ketosis prone diabetes during near-normoglycemia remission. Diabetes Care 2010; 33: 854-60.

16. Patel S, Hsu J, Jahoor F, Coraza I, Bain J, Stevens R, et al. Pathogenesis of A- $\beta+$ ketosis-prone diabetes. Diabetes 2013; 62: 912-22.

17. Umpierrez GE, Smiley D, Robalino G, Peng L, Gosmanov AR, Kitabchi AE. Lack of lipotoxicity effect on $\beta$ cell dysfunction in ketosis prone type 2 diabetes. Diabetes Care 2010; 33: 626-31.

18. Sobngwi E, Gautier JF, Kevorkian JP, Villette JM, Riveline JP, Zhang S, et al. High prevalence of glucose 6 phosphate deshydrogenase deficiency without gene mutations suggests a novel genetic mechanism predisponing to ketosis prone diabetes. J Clin Endocrinol Metab 2005; 90: 4446-51.

19. Choukem SP, Sobngwi E, Boudou P, Fetita L-S, Porcher $\mathrm{R}$, Ibrahim $\mathrm{F}$, et al. $\beta$ and $\alpha$ cell dysfunctions in Africans with ketosis prone atypical diabetes near normoglycemic remission. Diabetes Care 2013; 36: 118-23.

20. Kitabchi AE, Umpierrez GE, Miles JM, Fisher JN. Hyperglycemic crises in adult patients with diabetes. Diabetes Care 2009; 32: 1335-43.

21. Balasubramanyam A, Nalini R, Hampe CS, Gaur L, Maldonado M. Syndromes of ketosis-prone diabetes mellitus. Endocr Rev 2008; 29: 292-302. 